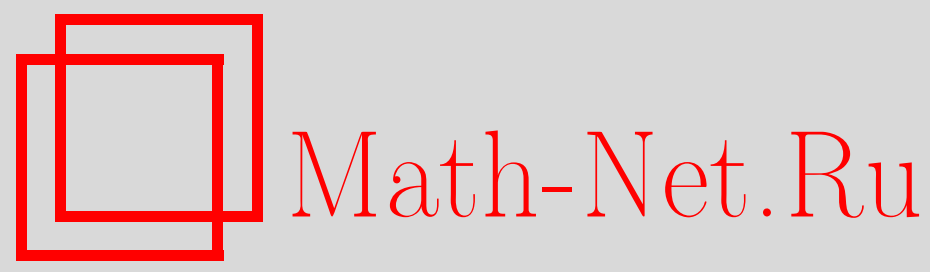

Е. С. Гарипова, Л. С. Казарин, О конечных почтикольцах, порожденных эндоморфизмами экстраспециальной 2группы, Дискрет. матем., 2010, том 22, выпуск 1, 104-114

DOI: https://doi.org/10.4213/dm1087

Использование Общероссийского математического портала Math-Net.Ru подразумевает, что вы прочитали и согласны с пользовательским соглашением http://www . mathnet.ru/rus/agreement

Параметры загрузки:

IP : 3.89 .197 .203

26 апреля 2023 г., 15:45:15 


\title{
О конечных почтикольцах, порожденных эндоморфизмами экстраспециальной 2-группы
}

\author{
() 2009 г. $\quad$ Е. С. Гарипова, Л. С. Казарин
}

\begin{abstract}
В статье рассматриваются почтикольца, порожденные эндоморфизмами некоторых экстраспециальных 2-групп. Наиболее существенным отличием почтикольца от обычного кольца является отсутствие второй дистрибутивности. Сформулированы и доказаны утверждения о том, что почтикольцо $E(G)$, порожденное эндоморфизмами экстраспециальной 2-группы $G$ порядка $2^{2 n+1}$, имеет порядок, делящий $2^{2^{2 n}+4 n^{2}}$, и о том, что почтикольцо $E(G)$ экстраспециальной 2-группы $G$ типа - порядка $2^{2 n+1}$ имеет порядок, делящийся на $2^{2^{2 n}+4 n^{2}-2}$. При этом для случаев $n=1$ и $n=2$ верхняя оценка достигается: так, почтикольцо $E(G)$ группы $D_{8}$ имеет порядок $2^{8}$, а почтикольцо $E(G)$ экстраспециальной 2-группы $D_{8} * Q_{8}$ имеет порядок, равный $2^{32}$.
\end{abstract}

\section{1. Введение}

Почтикольца - это множества с операциями сложения и умножения, удовлетворяющие законам кольца, за исключением коммутативности и одного из законов дистрибутивности. Мы будем использовать правую дистрибутивность.

Множество $M(G)$ всех отображений группы $G$ в себя является классическим примером почтикольца.

Впервые почтикольца появились в работе Диксона, который ввел понятие почтиполя. Первоначально такие структуры использовались в связи с их применением в геометрии. В конце тридцатых годов прошлого века ряд результатов в этом направлении был получен Виландом (не опубликовано). Теоретико-групповые аспекты почтиколец исследовались в конце пятидесятых годов Ханной Нейман. Позднее были открыты важные приложения почтиколец, в частности, к построениям блок-схем, группам перестановок и связанным с этим вопросам. Далее были найдены приложения в теории автоматов и в теории кодирования.

Первая монография, посвященная почтикольцам, опубликована Пильцем в 1977 г. В 1983 г. вышло второе издание [1], в котором содержался обзор важнейших результатов о почтикольцах и их приложениях. К настоящему времени опубликовано уже несколько монографий, в частности [2] и [3]. Книга [3], перевод которой [4] осуществлен в 1996 г., дает представление о некоторых приложениях почтиколец.

На сегодняшний день получены описания строения некоторых почтиколец небольшого порядка. В частности, Малон [8] описал почтикольцо, порожденное эндоморфизмами группы кватернионов. Как будет показано ниже, его результат для группы кватернионов порядка 8 неверен. Впрочем, работ, посвященных конкретным почтикольцам, немного. 
Следует отметить, что почтикольца, содержащиеся в кольце $M(G)$ всех отображений конечной группы $G$ в себя, являются естественным объектом в прикладной алгебре, где важными являются конструкции, связанные с различными отображениями группы в себя.

В. М. Сидельниковым была предложена идея использования почтиколец $E(G)$, порожденных эндоморфизмами небольшой конечной группы $G$, для изучения рекуррентных последовательностей. В этом случае необходимы знания о строении, именно, о порядке таких колец. И. В. Филимоновым, учеником В. М. Сидельникова, с помощью компьютера, найден порядок почтикольца эндоморфизмов группы диэдра порядка 8. Группы кватернионов и диэдра порядка 8 являются наименьшими в серии так называемых экстраспециальных $p$-групп. Последние довольно часто возникают в различных приложениях. Например, В. М. Сидельниковым [4] были открыты некоторые интересные свойства групп автоморфизмов экстраспециальных $p$-групп и построены на этой основе орбитные коды с хорошими характеристиками. Другое приложение, также найденное В. М. Сидельниковым [5], показало, что экстраспециальные 2-группы полезны для построения квантовых кодов.

Нами найдены порядки и строение почтиколец, порожденных эндоморфизмами экстраспециальных групп $G$ типа - порядков $2^{3}$ и $2^{5}$ (теоремы $1-3$ ), получена оценка порядка произвольной экстраспециальной 2-группы (теорема 4). Для экстраспециальной 2-группы порядка $2^{2 n+1}$ типа - эта оценка весьма близка к верхней (теорема 5). Вероятно, и это подтверждается для небольших групп, все булевы функции от $2 n$ переменных могут быть описаны как подпочтикольца почтикольца $E(G)$ экстраспециальной 2-группы $G$ порядка $2^{2 n+1}$.

Перейдем теперь к более формальным определениям и изложению результатов.

\section{2. Примеры почтиколец}

В статье рассматриваются алгебраические системы, удовлетворяющие следующему определению.

Почтикольцом называется алгебраическая система $R$ с двумя операциями, сложением и умножением, удовлетворяющая следующим условиям:

(i) $(R,+)$ - группа (не обязательно абелева);

(ii) $(R, \cdot)-$ полугруппа;

(iii) $(a+b) c=a c+b c$ для любых $a, b, c \in R$.

В этом случае почтикольцо называется правым.

Следующие примеры почтиколец являются классическими.

(1) Пусть $(G,+)$ - конечная (не обязательно абелева) группа. Тогда почтикольцом будет множество $M(G)=\left\{G^{G},+, \cdot\right\}$ всех отображений из $G$ в $G$ с операциями поточечного сложения (то есть $(f+g)(x)=f(x)+g(x))$ для любых двух отображений $f, g \in M(G)$ и любого $x \in G$ и суперпозицией отображений в качестве умножения (то есть $(f g)(x)=f(g(x)))$ для любых двух отображений $f, g \in M(G)$ и любого $x \in G$.

(2) Почти-кольцо $M_{0}(G)$, состоящее из всех $f \in M(G)$, для которых $f(0)=0$, и почти-кольцо констант $M_{c}(G)$, состоящее из отображений $f \in M(G)$, постоянных на $G$. 
(3) Пусть $V$ - линейное векторное пространство, тогда

$$
M_{\mathrm{Aff}}(V)=\{f \in M(V) \mid f(x)=A(x)+b\},
$$

где $A$ - линейное отображение, а $x, b \in V$, также будет почти-кольцом.

(4) Произвольная конечная группа $G$, имеющая порядок больший, чем 2 , может быть превращена в так называемое тривиальное почтикольцо путем введения операции умножения

$$
a \cdot b= \begin{cases}b, & \text { если } a \in S \subseteq G \backslash\{0\}, \\ 0 & \text { в противном случае. }\end{cases}
$$

\section{3. Некоторые определения и леммы}

Абелева $p$-группа называется гомоциклической, если она является прямым произведением своих циклических подгрупп одинакового порядка $p^{m}$. В случае $m=1$ группа называется элементарной. Если $p^{n}$ - это максимум порядков элементов $p$-группы, то говорят, что группа имеет экспоненту $p^{n}$. Таким образом, если абелева группа имеет экспоненту $p$, то она элементарна.

Пусть $\Phi(G)$ - подгруппа Фраттини группы $G$, то есть пересечение всех максимальных подгрупп группы $G$, если они существуют, и сама $G$ в противном случае.

Пусть $G^{\prime}$ - коммутант группы $G$, то есть подгруппа, которую образуют все коммутаторы группы.

Пусть $Z(G)$ - центр $G$, то есть подгруппа, которую образуют элементы, перестановочные со всеми элементами группы $G$.

Говорят, что $p$-группа $G$ специальна, если либо $G$ - элементарная абелева группа, либо ее коммутант $G^{\prime}$ совпадает с ее подгруппой Фраттини $\Phi(G)$ и с ее центром $Z(G)$ и является элементарной группой. Кроме того, $G$ называется экстраспециальной, если $G-$ неабелева специальная группа и $\left|G^{\prime}\right|=p$.

Группа $X$ называется центральным произведением групп $A$ и $B$, если $X=A B$ и $[A, B]=1$. Это произведение прямое, если $A \cap B=1$. Если же $A \cap B \neq 1$, то $A \cap B \leqslant Z(A) \cap Z(B)$. Для центрального произведения вводим обозначение $X=A * B$.

Пусть $Q_{8}$ и $D_{8}-$ кватернионная и диэдральная группы восьмого порядка, образующие элементы и определяющие соотношения которых будут подробно рассмотрены ниже.

Если $G$ - экстраспециальная $p$-группа, то $G=A_{1} * A_{2} * \ldots * A_{n}$, где $A_{i}, i=1, \ldots, n$, - экстраспециальная группа порядка $p^{3}$ (см. [6]). Целое число $n$ называется шириной экстраспециальной группы $G$. Для заданного $p$ имеется ровно две неизоморфные экстраспециальные $p$-группы порядка $p^{3}$. В случае $p=2$ соответствующие группы - это кватернионная группа порядка 8 и диэдральная группа порядка 8 , причем $Q_{8} * Q_{8} \cong D_{8} * D_{8}$. Поэтому, если $G$ - экстраспециальная 2-группа ширины $n$, то $G$ изоморфна $\left(Q_{8}\right)^{n}$ или $\left(Q_{8}\right)^{n-1} * D_{8}$. В первом случае, говорят, что $G-$ это группа типа + , во втором - группа типа -.

Предположим, что $G$ - экстраспециальная группа, $Z(G)=\langle z\rangle$ и $\bar{G}=G / Z(G)$. Тогда $\bar{G}$ - векторное пространство над $G F(p)$ и $|z|=p$.

Пусть $M_{2 n}(G F(2))$ - полное матричное кольцо квадратных $2 n$-строчных матриц над полем Галуа $G F(2)$ и $O_{2 n}^{ \pm}(2)$ - ортогональная группа четной размерности над полем из двух элементов.

Пусть $\operatorname{End}(G) \subseteq M(G)-$ множество эндоморфизмов группы $G$. Сумма двух эндоморфизмов, вообще говоря, не является эндоморфизмом. Поэтому обозначим через $E(G)$ 
наименьшее подпочтикольцо $M(G)$, содержащее $\operatorname{End}(G)$, то есть почтикольцо, порожденное эндоморфизмами группы $G$.

Лемма 1. Пусть $G-$-группа с коммутантом порядка $p$, тогда для любых $\varphi \in E(G)$, $a \in G, z \in G^{\prime}$

$$
\varphi(a+z)=\varphi(a)+\varphi(z) .
$$

Доказательство. По определению любой элемент из $E(G)$ есть целочисленная линейная комбинация эндоморфизмов $\operatorname{End}(G)$ из множества, поэтому

$$
\varphi=\sum_{i=1}^{k} \lambda_{i} \psi_{i}
$$

где $\lambda_{i}$ - целые числа (на деле они ограничены по модулю экспоненты группы $G$ ). Тогда для любого $a \in G$ и любого $z \in G^{\prime}$

$$
\varphi(a+z)=\left(\sum_{i=1}^{k} \lambda_{i} \psi_{i}\right)(a+z)=\sum_{i=1}^{k} \lambda_{i} \psi_{i}(a+z)=\sum_{i=1}^{k} \lambda_{i}\left(\psi_{i}(a)+\psi_{i}(z)\right) .
$$

Так как $\psi_{i}(z)$ содержится в центре $G$ для любого $z \in G^{\prime}$, легко видеть, что последнее выражение совпадает с

$$
\sum_{i=1}^{k} \lambda_{i} \psi_{i}(a)+\sum_{i=1}^{k} \lambda_{i} \psi_{i}(z)=\varphi(a)+\varphi(z) .
$$

Почтикольцо $I$ называется идеалом почтикольца $R$, если выполнены следующие условия:

(i) $I^{+}-$нормальная подгруппа группы $R^{+}$;

(ii) для любых $a \in I, r \in R$ справедливо включение $a r \in I$;

(iii) для любых $a \in I, r, s \in R$ справедливо включение $s(r+a)-s r \in I$.

Лемма 2. Если $K$ - вполне характеристическая подгруппа группь $G$, то

$$
S=\{f \in E(G) \mid f(x) \in K, x \in G\}
$$

является идеалом $R=E(G)$.

Доказательство. Пусть $K$ - вполне характеристическая подгруппа группы $G$. Покажем, что множество $f \in E(G)$, отправляющих любой элемент из $G$ в $K$, составляет идеал $S$ в $R$. В самом деле, если $f(x) \in K$, то, в силу нормальности характеристической подгруппы,

$$
(-g+f+g)(x)=-g(x)+f(x)+g(x) \in K
$$

для любого $g \in E(G)$. Замкнутость доказана. Поэтому $S^{+}-$нормальная подгруппа $R^{+}$.

Пусть $f \in S, r \in R$. Тогда

$$
(f r)(x)=f(r(x)) \in K
$$

для любого $x \in G$. Следовательно, $f r \in S$ для любого $f \in S$. 
Покажем, что $f(r+s)-f r \in S$ для любых $r, f \in R, s \in S$. Действительно, если $f(x+y)=f(x)+f(y)$ (например, это так, если $f$ - эндоморфизм), то указанный элемент принадлежит $S$ ввиду ее инвариантности как подгруппы аддитивной группы $R^{+}$. В самом деле, если $x \in G$, то

$$
f(r+s)(x)-f r(x)=f(r(x)+s(x))-f r(x) .
$$

Если $f$ - эндоморфизм, то результат содержится в $K$. Если же $f$ - сумма или произведение двух элементов $E(G)$, то применим индукцию. Пусть $f=f^{\prime}+f^{\prime \prime}$, где $f^{\prime} \in \operatorname{End}(G)$, а элемент $f^{\prime \prime}$ такой, что $f^{\prime \prime}(r+s)(x)-f^{\prime \prime} r(x) \in S$, тогда

$$
\left(f^{\prime}+f^{\prime \prime}\right)(r+s)-\left(f^{\prime}+f^{\prime \prime}\right) r=f^{\prime}(r+s)+f^{\prime \prime}(r+s)-f^{\prime} r-f^{\prime \prime} s .
$$

Используя нормальность подгруппы $S^{+}$в $R^{+}$, получаем по индукции справедливость соотношения $f(r+s)-f r \in S$. Аналогичным образом можно рассмотреть ситуацию, когда $f=f^{\prime} f^{\prime \prime}$, где один из сомножителей является эндоморфизмом.

\section{4. Основные результаты}

Теорема 1. Почтикольцฺо $E(G)$ экстраспециальной 2-группь $D_{8}$ имеет порядок, равный̌ $2^{8}$.

Доказательство. Здесь $G=D_{8},|G|=8$, определяющими в группе диэдра являются соотношения $a^{4}=1, b^{2}=1, b^{-1} a b=a^{-1}$.

Идеал $J$, состоящий из отображений в $E(G)$, посылающих любой элемент $D_{8}$ в элементы $z=2 a$ или 0 , состоит из 0 и отображений, тождественно равных 0 на $G / Z(G)$. В силу леммы 1 , знание этих отображений на элементах, являющихся представителями левых смежных классов по подгруппе $Z=G^{\prime}=Z(G)$, и на $z$ позволяет вычислить значение на любом элементе группы. Один из представителей является нулем группы, и значение любого эндоморфизма из $\operatorname{End}(G)$ на этом элементе равно 0. Остальные элементы из системы представителей (их всего три), вместе с элементом $z$, являющимся единственным ненулевым элементом из $Z$, определяют любое отображение из $E(G)$, лежащее в $J$. Более того, все такие отображения образуют линейное векторное пространство над полем $G F(2)$, ибо экспонента группы $Z$ равна двум. Таким образом, число таких отображений не превосходит $2^{4}$.

Покажем, что имеется 4 линейно независимых над $G F(2)$ функций, лежащих в $E(G)$ и отображающих $G$ в $Z$. Кроме того, покажем, что фактор-почтикольцо $E(G) / J$ тоже состоит из $2^{4}$ элементов.

Группа $G$ имеет эндоморфизмы $\eta_{1}, \eta_{2}, \eta_{3} \in \operatorname{End}(G)$ :

$$
\begin{array}{lll}
\eta_{1}(a)=0, & \eta_{1}(b)=z, & \eta_{1}(a+b)=z ; \\
\eta_{2}(a)=z, & \eta_{2}(b)=0, & \eta_{1}(a+b)=z ; \\
\eta_{3}(a)=z, & \eta_{3}(b)=z, & \eta_{3}(a+b)=0 .
\end{array}
$$

При этом очевидно, что $\eta_{1}+\eta_{2}+\eta_{3}=0$. Кроме того, преобразования $\eta_{j}$ оказываются нильпотентными индекса $2: \eta_{j}^{2}=0$ для любого $j=1,2,3$. 
Кроме того, имеются эндоморфизмы $\psi_{1}, \psi_{2}$ и $\psi_{3}$, действующие следующим образом на группе $G$ :

$$
\begin{array}{lll}
\psi_{1}(a)=b, & \psi_{1}(b)=0, & \psi_{1}(a+b)=b ; \\
\psi_{2}(a)=a+b, & \psi_{2}(b)=0, & \psi_{2}(a+b)=a+b ; \\
\psi_{3}(a)=0, & \psi_{3}(b)=b, & \psi_{3}(a+b)=b .
\end{array}
$$

Обозначим через $i$ тождественный автоморфизм группы $G$, а через $\theta$ линейную комбинацию $i+\psi_{1}+\psi_{2}+\psi_{3}$.

Получаем таблицу значений

\begin{tabular}{c|cccc} 
& $a$ & $b$ & $a+b$ & $z$ \\
\hline $2 i$ & $z$ & 0 & 0 & 0 \\
$\eta_{3}$ & $z$ & $z$ & 0 & 0 \\
$\eta_{1}$ & 0 & $z$ & $z$ & 0 \\
$\theta$ & 0 & 0 & 0 & $z$
\end{tabular}

Таким образом, имеем линейно независимую систему элементов почтикольца, содержащихся в $J:\left\{\eta_{1}, \eta_{3}, 2 i, \theta\right\}$, то есть $(J,+)$ - элементарная абелева группа порядка $2^{4}$.

Элементы $\psi_{1}, \psi_{2}, \psi_{3}$, определенные выше, вместе с автоморфизмом $i$, будут являться элементами, индуцирующими на $G / Z$ линейно независимую систему преобразований.

Значит, $|E(G)|=2^{4} 2^{4}=2^{8}$. Следует заметить, что это согласуется с результатом И. В. Филимонова, который при помощи компьютера показал, что $\left|E\left(D_{8}\right)\right|=256$.

Примечательно, что такую же размерность имеет почтикольцо, порожденное эндоморфизмами группы $Q_{8}$, экстраспециальной 2-группы типа + .

Теорема 2. Почтикольц̧о $E(G)$ экстраспецииальной 2-группь $Q_{8}$ имеет порядок, равнызй $2^{8}$.

Доказательство. Здесь $G=Q_{8},|G|=8$, определяющими в группе кватернионов являются соотношения $a^{4}=1 ; b^{4}=1 ; a^{2}=b^{2} ; a b a=b$.

Доказательство проводим аналогично случаю типа -. Идеал $J$, состоящий из отображений в $E(G)$, посылающих любой элемент $G=Q_{8}$ в элементы $2 a=z$ или 0 , состоит из 0 и отображений, тождественно равных 0 на $G / Z(G)$. В силу леммы 1 , знание этих отображений на элементах, являющихся представителями левых смежных классов по подгруппе $Z=G^{\prime}=Z(G)$, и на $z$ позволяет вычислить значение на любом элементе группы. Один из представителей является нулем группы, и значение любого эндоморфизма из $\operatorname{End}(G)$ на этом элементе равно 0. Остальные элементы из системы представителей (их всего три), вместе с элементом $z$, являющимся единственным ненулевым элементом из $Z$, определяют любое отображение из $E(G)$, лежащее в $J$. Более того, все такие отображения образуют линейное векторное пространство над полем $G F(2)$, ибо экспонента группы $Z$ равна двум. Таким образом, число таких отображений не превосходит $2^{4}$.

Покажем, что имеется 4 линейно независимых над $G F(2)$ функций, лежащих в $E(G)$ и отображающих $G$ в $Z$. Кроме того, покажем, что фактор-почтикольцо $E(G) / J$ тоже состоит из $2^{4}$ элементов.

Группа $G$ имеет эндоморфизмы $\eta_{1}, \eta_{2}, \eta_{3} \in \operatorname{End}(G)$ :

$$
\begin{array}{lll}
\eta_{1}(a)=0, & \eta_{1}(b)=z, & \eta_{1}(a+b)=z ; \\
\eta_{2}(a)=z, & \eta_{2}(b)=0, & \eta_{1}(a+b)=z ; \\
\eta_{3}(a)=z, & \eta_{3}(b)=z, & \eta_{3}(a+b)=0 .
\end{array}
$$


При этом очевидно, что $\eta_{1}+\eta_{2}+\eta_{3}=0$.

Кроме того, $G$ имеет автоморфизм $\varphi$, действующий на элементах $G$ следующим образом:

$$
\varphi(a)=b, \quad \varphi(b)=a+b, \quad \varphi(a+b)=a .
$$

Положим $\theta=i+\varphi+\varphi^{2}$, где $i-$ тождественный автоморфизм. Тогда получим следующую систему элементов почтикольца, содержащихся в $J:\left\{2 \varphi+\eta_{1}, 2 \varphi+\eta_{2}, 2 \varphi+\eta_{3}, \theta\right\}$. Линейная независимость указанных элементов $J$ следует из таблицы

\begin{tabular}{c|cccc} 
& $a$ & $b$ & $a+b$ & $z$ \\
\hline $2 \varphi+\eta_{1}$ & $z$ & 0 & 0 & 0 \\
$2 \varphi+\eta_{2}$ & 0 & $z$ & 0 & 0 \\
$2 \varphi+\eta_{3}$ & 0 & 0 & $z$ & 0 \\
$\theta$ & $z$ & $z$ & $z$ & $z$
\end{tabular}

Таким образом, $(J,+)$ - элементарная абелева группа порядка $2^{4}$. Каждый эндоморфизм группы $G$ индуцирует линейное преобразование на факторгруппе $G / Z(G)$, изоморфной четверной группе Клейна. При этом возможно любое из $2^{4}$ таких линейных преобразований. Поэтому общее число элементов в $E(G)$ оказывается равным $2^{8}$.

Отметим, что в статье [7] утверждается, что почтикольцо $E(G)$ группы кватернионов порядка 8 состоит из $2^{5}$ элементов. Из теоремы 2 следует, что это утверждение ошибочно.

Перейдем к случаю $n=2$.

Теорема 3. Почтикольияо $E(G)$ экстраспецииальной 2-группь $D_{8} * Q_{8}$ имеет порядок, равный $2^{32}$.

Доказательство. Пусть $G$ - экстраспециальная 2-группа ширины 2 типа -. Тогда она будет являться центральным произведением группы кватернионов и группы диэдра. Далее, $D_{8}$ и $Q_{8}$ имеют одинаковые порядки, равные 8 , и состоят из элементов вида $a^{s} b^{t}$, где $0 \leqslant s \leqslant 3,0 \leqslant t \leqslant 1$.

Определяющие соотношения в группах кватернионов и диэдра приведены в предыдущих теоремах. При рассмотрении этого случая будет удобно пользоваться следующей таблицей порядков элементов групп $D_{8}$ и $Q_{8}$ :

\begin{tabular}{c|cc} 
& $D_{8}$ & $Q_{8}$ \\
\hline$e$ & 1 & 1 \\
$a$ & 4 & 4 \\
$b$ & 2 & 4 \\
$a^{2}$ & 2 & 2 \\
$a^{3}$ & 4 & 4 \\
$a b$ & 2 & 4 \\
$a^{2} b$ & 2 & 4 \\
$a^{3} b$ & 2 & 4
\end{tabular}

Склеивать группы будем по центрам - циклическим подгруппам второго порядка:

$$
\begin{gathered}
Z\left(D_{8}\right) \cong Z\left(Q_{8}\right) \cong\left\langle a^{2}\right\rangle ; \quad G=D_{8} * Q_{8} ; \\
|G|=(8 \cdot 8) / 2=32, \quad|Z(G)|=\left|G^{\prime}\right|=2 ; \\
|G / Z(G)|=32 / 2=2^{4}=16 .
\end{gathered}
$$


Таблица 1.

\begin{tabular}{c|cccccccccccccccc} 
& $\alpha_{1}$ & $\alpha_{2}$ & $\alpha_{3}$ & $\alpha_{4}$ & $\alpha_{5}$ & $\alpha_{6}$ & $\alpha_{7}$ & $\alpha_{8}$ & $\alpha_{9}$ & $\alpha_{10}$ & $\alpha_{11}$ & $\alpha_{12}$ & $\alpha_{13}$ & $\alpha_{14}$ & $\alpha_{15}$ & $z$ \\
\hline$\varphi_{1}$ & $z$ & $z$ & $z$ & $z$ & $z$ & $z$ & $z$ & $z$ & 0 & 0 & 0 & 0 & 0 & 0 & 0 & 0 \\
$\varphi_{2}$ & 0 & $z$ & $z$ & $z$ & $z$ & $z$ & $z$ & $z$ & $z$ & 0 & 0 & 0 & 0 & 0 & 0 & 0 \\
$\varphi_{3}$ & 0 & 0 & $z$ & $z$ & $z$ & $z$ & $z$ & $z$ & $z$ & $z$ & 0 & 0 & 0 & 0 & 0 & 0 \\
$\varphi_{4}$ & 0 & 0 & 0 & $z$ & $z$ & $z$ & $z$ & $z$ & $z$ & $z$ & $z$ & 0 & 0 & 0 & 0 & 0 \\
$\varphi_{5}$ & 0 & 0 & 0 & 0 & $z$ & $z$ & $z$ & $z$ & $z$ & $z$ & $z$ & $z$ & 0 & 0 & 0 & 0 \\
$\varphi_{6}$ & 0 & 0 & 0 & 0 & 0 & $z$ & $z$ & $z$ & $z$ & $z$ & $z$ & $z$ & $z$ & 0 & 0 & 0 \\
$\varphi_{7}$ & 0 & 0 & 0 & 0 & 0 & 0 & $z$ & $z$ & $z$ & $z$ & $z$ & $z$ & $z$ & $z$ & 0 & 0 \\
$\varphi_{8}$ & 0 & 0 & 0 & 0 & 0 & 0 & 0 & $z$ & $z$ & $z$ & $z$ & $z$ & $z$ & $z$ & $z$ & 0 \\
$\varphi_{9}$ & $z$ & 0 & 0 & 0 & 0 & 0 & 0 & 0 & $z$ & $z$ & $z$ & $z$ & $z$ & $z$ & $z$ & 0 \\
$\varphi_{10}$ & $z$ & $z$ & 0 & 0 & 0 & 0 & 0 & 0 & 0 & $z$ & $z$ & $z$ & $z$ & $z$ & $z$ & 0 \\
$\varphi_{11}$ & $z$ & $z$ & $z$ & 0 & 0 & 0 & 0 & 0 & 0 & 0 & $z$ & $z$ & $z$ & $z$ & $z$ & 0 \\
$\varphi_{12}$ & $z$ & $z$ & $z$ & $z$ & 0 & 0 & 0 & 0 & 0 & 0 & 0 & $z$ & $z$ & $z$ & $z$ & 0 \\
$\varphi_{13}$ & $z$ & $z$ & $z$ & $z$ & $z$ & 0 & 0 & 0 & 0 & 0 & 0 & 0 & $z$ & $z$ & $z$ & 0 \\
$\varphi_{14}$ & $z$ & $z$ & $z$ & $z$ & $z$ & $z$ & 0 & 0 & 0 & 0 & 0 & 0 & 0 & $z$ & $z$ & 0 \\
$\varphi_{15}$ & $z$ & $z$ & $z$ & $z$ & $z$ & $z$ & $z$ & 0 & 0 & 0 & 0 & 0 & 0 & 0 & $z$ & 0 \\
$\varkappa$ & 0 & 0 & $z$ & $z$ & $z$ & $z$ & 0 & $z$ & 0 & $z$ & 0 & $z$ & $z$ & $z$ & 0 & 0 \\
$\zeta$ & 0 & 0 & 0 & 0 & 0 & 0 & 0 & 0 & 0 & 0 & 0 & 0 & 0 & 0 & 0 & $z$
\end{tabular}

Представители смежных классов $G$ по $Z$ в аддитивной записи выглядят следующим образом:

$$
\begin{aligned}
& \alpha_{1}=\left(0, a_{2}\right) \text {, } \\
& \left(a_{1}, b_{2}\right)=\alpha_{6}, \\
& \left(b_{1}, a_{2}+b_{2}\right)=\alpha_{11} \text {, } \\
& \alpha_{2}=\left(0, b_{2}\right) \text {, } \\
& \left(a_{1}, a_{2}+b_{2}\right)=\alpha_{7} \text {, } \\
& \left(a_{1}+b_{1}, 0\right)=\alpha_{12} \text {, } \\
& \alpha_{3}=\left(0, a_{2}+b_{2}\right) \text {, } \\
& \left(b_{1}, 0\right)=\alpha_{8} \text {, } \\
& \left(a_{1}+b_{1}, a_{2}\right)=\alpha_{13} \text {, } \\
& \alpha_{4}=\left(a_{1}, 0\right) \text {, } \\
& \left(b_{1}, a_{2}\right)=\alpha_{9} \text {, } \\
& \left(a_{1}+b_{1}, b_{2}\right)=\alpha_{14} \text {, } \\
& \alpha_{5}=\left(a_{1}, a_{2}\right) \text {, } \\
& \left(b_{1}, b_{2}\right)=\alpha_{10}, \quad\left(a_{1}+b_{1}, a_{2}+b_{2}\right)=\alpha_{15} \text {. }
\end{aligned}
$$

Далее будет показано, что имеется $2^{2 n}$ линейно независимых над $G F(2)$ функцийэндоморфизмов, лежащих в $E(G)$ и отображающих $G$ в $Z$.

Элемент $z$ содержится в ядре любого такого эндоморфизма. Образ его - циклическая подгруппа второго порядка, а ядро - абелева подгруппа группы $G$ индекса 2. Число таких подгрупп в $G$ равно

$$
\frac{p^{2 n}-1}{p-1}=\frac{2^{2 \cdot 2}-1}{2-1}=2^{4}-1=15 .
$$

Следовательно, имеется 15 ненулевых эндоморфизмов $\varphi_{1}, \ldots \varphi_{15}$ таких что

$$
\left|\operatorname{Ker}\left(\varphi_{i}\right)\right|=8, \quad \varphi_{i}\left(\alpha_{j}\right) \text { равно } z \text { или } 0, \quad \varphi_{i}^{2}\left(\alpha_{j}\right)=0, \quad \sum_{i=1}^{15} \varphi_{i}=0 .
$$

Действие их представлено в табл. 1.

Каждый эндоморфизм группы $G$ индуцирует линейное преобразование на элементарной абелевой фактор-группе $G / Z(G)$. Будем считать ее линейным векторным пространством по сложению над $G F(2)$, оснащенным билинейной формой

$$
x Z+y Z=(x+y) Z \text {. }
$$


Как симметрическую билинейную форму над $G F(2)$ будем трактовать операцию взятия коммутатора:

$$
\begin{gathered}
\mathscr{A}(x, y)=[x, y], \quad \mathscr{A}(\alpha x, y)=\alpha \mathscr{A}(x, y), \quad \mathscr{A}(x, \alpha y)=\alpha \mathscr{A}(x, y), \\
\mathscr{A}(x+y, z)=\mathscr{A}(x, z)+\mathscr{A}(y, z), \quad \mathscr{A}(x, y+z)=\mathscr{A}(x, y)+\mathscr{A}(x, z), \\
\mathscr{A}(x, x)=0 \text { since }[x, x]=x x^{-1} x x^{-1}=1, \quad \mathscr{A}(x, y)=[x, y]=[y, x]=\mathscr{A}(y, x) .
\end{gathered}
$$

Получаем пространство четной размерности над полем характеристики 2.

Убедимся далее, что в определенном нами линейном пространстве сохраняется квадратичная форма.

Для этого более удобно воспользоваться копредставлением диэдральной группы, поэтому пусть теперь $d_{1}, d_{2}-$ ее инволютивные образующие, $d_{1}=a_{1} b_{1}, d_{2}=b_{1}$, тогда

$$
\begin{aligned}
{\left[d_{1}, d_{2}\right] } & =1, & {\left[a_{2}, b_{2}\right] } & =1, \\
{\left[d_{1}, a_{2}\right]=\left[d_{1}, b_{2}\right] } & =0, & {\left[d_{2}, a_{2}\right]=\left[d_{2}, b_{2}\right] } & =0 .
\end{aligned}
$$

Любой элемент $g$ из $G$ представим в виде линейной комбинации базисных элементов:

$$
g=x_{1} d_{1}+x_{2} d_{2}+x_{3} a_{2}+x_{4} b_{2} .
$$

Зададим на линейном пространстве квадратичную форму

$$
Q(g)=x_{1} x_{2}+x_{3}^{2}+x_{3} x_{4}+x_{4}^{2} .
$$

Очевидно, что элемент $g$ можно представить в виде суммы двух компонент $a \in\left\langle d_{1}, d_{2}\right\rangle$ и $b \in\left\langle a_{2}, b_{2}\right\rangle$. Тогда $x_{1} x_{2}-$ это вклад элемента $a$ в квадратичную форму, а $x_{3}^{2}+x_{3} x_{4}+x_{4}^{2}$ - вклад $b$.

Если $Q(a)=1$, а это возможно только когда $x_{1}=x_{2}=1$, то $Q(g)=Q(a+b)=0$ при любом $b \neq 0$. Если же $b=0$, то $Q(g)=1$.

В случае, когда $Q(a)=0$ (варианты: $x_{1}=x_{2}=0 ; x_{1}=1, x_{2}=0$ и $x_{1}=0$, $\left.x_{2}=1\right)$, для сохранения квадратичной формой нулевого значения необходимо, чтобы $b$ было равно 0.

Получаем пять ненулевых элементов, на которых квадратичная форма обращается в 0:

$$
\begin{gathered}
(1,0,0,0)=d_{1}, \quad(0,1,0,0)=d_{2}, \quad(1,1,1,0)=d_{1}+d_{2}+a_{2}, \\
(1,1,0,1)=d_{1}+d_{2}+b_{2}, \quad(1,1,1,1)=d_{1}+d_{2}+a_{2}+b_{2} .
\end{gathered}
$$

Заметим, что эти элементы в группе $G$ имеют порядок, равный 2. Построим автоморфизм $\theta$, циклически сдвигающий найденные инволютивные элементы:

$$
\begin{gathered}
\theta\left(d_{1}\right)=d_{2}, \quad \theta\left(d_{2}\right)=d_{1}+d_{2}+a_{2}, \quad \theta\left(d_{1}+d_{2}+a_{2}\right)=d_{1}+d_{2}+b_{2}, \\
\theta\left(d_{1}+d_{2}+b_{2}\right)=d_{1}+d_{2}+a_{2}+b_{2}, \quad \theta\left(d_{1}+d_{2}+a_{2}+b_{2}\right)=d_{1} .
\end{gathered}
$$

Этот автоморфизм, имеющий порядок, равный 5, будет сохранять заданную квадратичную форму.

В выбранном базисе его матрица имеет следующий вид:

$$
A=\left(\begin{array}{llll}
0 & 1 & 0 & 0 \\
0 & 0 & 1 & 0 \\
0 & 0 & 0 & 1 \\
1 & 1 & 1 & 1
\end{array}\right) .
$$


Матрица билинейной формы, составленная из скалярных произведений соответствующих базисных элементов, выглядит так:

$$
\Phi=\left(\begin{array}{llll}
0 & 1 & 1 & 1 \\
1 & 0 & 1 & 1 \\
1 & 1 & 0 & 1 \\
1 & 1 & 1 & 0
\end{array}\right) .
$$

Нетрудно проверить, что соотношение $A \Phi A^{-1}=\Phi$ выполнено, а значит, автоморфизм сохраняет билинейную форму.

Пусть $\zeta-$ эндоморфизм такой, что

$$
\zeta=1+\theta+\theta^{2}+\theta^{3}+\theta^{4},
$$

$\varkappa=2 \gamma+\delta-$ тоже эндоморфизм, где

$$
\begin{gathered}
\gamma\left(a_{i}\right)=b_{i}, \quad \gamma\left(b_{i}\right)=a_{i}+b_{i}, \quad \gamma\left(a_{i}+b_{i}\right)=a_{i}, \\
\delta\left(a_{i}\right)=\delta\left(b_{i}\right)=z, \quad \delta\left(a_{i}+b_{i}\right)=0, \quad i=1,2 .
\end{gathered}
$$

Таким образом, система $\left\{\varphi_{1}, \ldots \varphi_{14}, \varkappa, \zeta\right\}$ предоставляет $2^{2 n}$, в нашем случае 16 , ненулевых элементов, образующих линейно независимую систему преобразований, тождественно равных 0 на $G / Z(G)$. Поэтому порядок почтикольца может быть подсчитан как

$$
|E(G)|=|I||E(G) / I|=2^{16} \cdot 2^{16}=2^{32} .
$$

В общем случае, почтикольцо экстраспециальной группы можно рассматривать как расширение подпочтикольца $I$, состоящего из отображений, переводящих каждый элемент группы $G$ в элемент из подгруппы $Z(G)$ при помощи полного матричного кольца $M_{2 n}(G F(2))$.

Теорема 4. Почтикольйо $E(G)$ экстраспецииальной 2-группы $G$ порядка $2^{2 n+1}$ имеет порядок, делящчий $2^{2^{2 n}+4 n^{2}}$.

Доказательство. Пусть $G$ - экстраспециальная группа, указанного порядка.

Идеал $J$, состоящий из отображений в $E(G)$, посылающих любой элемент $G$ в элементы центра, состоит из 0 и отображений, тождественно равных 0 на $G / Z(G)$. В силу леммы 1 , знание этих отображений на элементах, являющихся представителями левых смежных классов по подгруппе $Z=G^{\prime}=Z(G)$, и на $z$ позволяет вычислить значение на любом элементе группы.

Один из представителей является нулем группы, и значение любого эндоморфизма из $\operatorname{End}(G)$ на этом элементе равно 0. Остальные элементы из системы представителей, вместе с элементом $z$, являющимся единственным ненулевым элементом из $Z$, определяют любое отображение из $E(G)$, лежащее в $J$, то есть имеется $2^{2 n}$ эндоморфизмов, тождественно равных 0 на $G / Z(G)$, определенных соотношениями $\varphi_{i}(x)=0$ для $x \in K$, где $K-$ подгруппа индекса два из $G$ и $\varphi_{i}(x)=z$ для $x \notin K$. При этом очевидно, что $\left(\varphi_{j}\right)^{2}=0$ для всех $j$. Все эти эндоморфизмы нильпотентны индекса 2 и по сложению образуют элементарную абелеву группу порядка $2^{2 n}$, а также идеал $J$ почтикольца $E(G)$.

Каждый эндоморфизм группы $G$ индуцирует линейное преобразование на факторгруппе $G / Z(G)$, изоморфной элементарной абелевой 2-группе порядка $2^{2 n}$. Так как группа автоморфизмов экстраспециальной 2 -группы порядка $2^{2 n+1}$ изоморфна $O_{2 n}^{ \pm}(2)$ и по 
следствию 1 из [9], с. 143, неприводима при $n>1$, линейная оболочка этих автоморфизмов в ограничении на $G / Z(G)$ изоморфна полному матричному кольцу $\mu_{2 n}(2)$, а значит, на фактор-группе может быть реализовано любое из $2^{(2 n)^{2}}$ линейных преобразований.

Другими словами, каждому отображению $f \in E(G)$ ставим в соответствие его ограничение $f^{\prime} \in E(G / Z(G))$. В случае, когда два различных преобразования в $E(G)$ индуцируют одно и то же линейное преобразование $G / Z(G)$, их разность есть преобразование из $E(G)$, переводящее любой элемент из $G$ в $Z(G)$. Поэтому общее число преобразований равно $|J| \mid E(G / Z(G) \mid$.

Таким образом, общее число элементов почтикольца равно $2^{2^{2 n}+4 n^{2}}$.

Нас интересует случай группы типа -, являющейся центральным произведением группы диэдра и некоторого числа групп кватернионов. Дело в том, что в этом случае система из $2^{2 n}$ эндоморфизмов идеала, описанных выше, оказывается зависимой. Максимальная линейно независимая подсистема, которую можно из них выделить, имеет ранг, равный $2^{2 n}-2$. Поэтому справедливо следующее утверждение.

Теорема 5. Почтикольияо $E(G)$ экстраспециальной 2-группь $G$ порядка $2^{2 n+1}$ типа имеет порядок, делящчийся на $2^{2^{2 n}+4 n^{2}-2}$.

Однако, как показано выше, для случаев $n=1$ и $n=2$ верхняя оценка все-таки достигается, то есть указан способ построения пары дополнительных эндоморфизмов, которые, вкупе с $2^{2 n}-2$ найденными, образуют полную линейно независимую систему из $2^{2 n}$ функций, лежащих в $E(G)$ и отображающих $G$ в $Z$. Это позволяет предполагать, что подобная пара найдется и для групп типа - большей ширины. Вопрос, верно ли, что $|E(G)|=2^{2^{2 n}+4 n^{2}}$ для экстраспециальной 2-группы $G$ в общем случае, остается открытым.

\section{Список литературы}

1. Pilz G., Near-rings. The theory and its applications. North-Holland, Amsterdam, 1983.

2. Meldrum J. D. P., Near-rings and their links with groups. Pitman, London, 1985.

3. Lidl R., Pilz G., Applied abstract algebra. Springer, New York, 1984.

4. Лидл Р., Пильц Г., Прикладная абстрактная алгебра. Изд-во Уральского университета, Екатеринбург, 1996.

5. Сидельников В. М., Об одной конечной матричной группе и кодах на евклидовой сфере. Проблемы передачи информации (1997) 33, №1, 35-54.

6. Сидельников В. М., Квантовые коды и абелевы подгруппы экстраспециальной группы. Проблемы передачи информации (2002) 38, №3, 34-44.

7. Gorenstein D., Finite simple groups. An introduction to their classification. Plenum, New York, 1982.

8. Malone J. J., Generalised quaternion groups and distributively generated near-rings. Proc. Edinb. Math. Soc., II Ser. (1973) 18, 235-238.

9. Дьедонне Ж., Геометрия классических групп. Мир, Москва, 1974.

10. Супруненко Д. А., Группь матрии. Наука, Москва, 1972.

Статья поступила 28.07.2009. 